\title{
Specific Differences in the Inhibition Titers of the Anti-H Lectins from Cytisus sessilifolius and Ulex europaeus *
}

\author{
Chris C. Plato and Henry Gershowitz
}

\begin{abstract}
University of Michigan Medical School, Department of Human Genetics Ann Arbor, Michigan
\end{abstract}

In 1948 Renkonen [9], and a year later, Boyd and Reguera [3] recognized that several plant seed extracts (lectins) act as haemagglutinins. Since that time the haemagglutinating action of the lectins has been verified by many investigators (see Bird [2]). It has also been demonstrated that some lectins are quite specific in their reactions with certain blood group substances (Bird [1], Boyd and Shapleigh [5], and Cazal and Lalaurie [6]).

The anti-H lectins are of special interest among these plant extracts, because of their usefulness in the determination of the secretor status where soluble blood group substances are present in the saliva (Boyd and Shapleigh [4]).

It is generally believed (see Glynn and Holborow [7]) that all secretors contain in their salivas detectable amounts of $H$ substance. Some exceptions to this generality were reported by $\mathrm{McNeil}$ et al. [8] who, by using Lotus tetragonolobus as an anti-H source, encountered some secretors whose saliva inhibited either the anti-A or the anti-B but not the anti-H. These cases they labelled as "aberrant secretors".

In addition to Lotus tetragonolobus, seed from Laburnum alpinum, Cytisus sessilifolius, Ulex europaeus, and Tetragonolobus purpureus have been commonly used as sources of anti-H (see Cazal and Lalaurie [6]). The objectives of this investigation were: (1) to determine whether any significant differences exist between the inhibition titers of the anti-H reagents obtained from seeds of two of these plants, when tested against the same saliva; (2) to establish whether salivas from secretors of different blood groups show differences in the inhibition titers in respect to these two lectins. To this end, the inhibition titers of the salivas of persons of $A_{1}, A_{2}, O, B$ and $A B$ blood groups were tested against constant dilutions of Cytisus sessilifolius and Ulex europaeus extracts.

* This investigation was supported by grants from the Kellogg Foundation and the United States Atomic Energy Commission (project AT (11-1)-405). 


\section{Materials, Data and Methods}

Material: The seeds used in this experiment were kindly supplied by the Botanical Gardens of the University of Lisbon, Portugal.

Reagents: Both the Cytisus sessilifolius and the Ulex europaeus anti-H reagents (which will be abbreviated as anti- $\mathrm{H}_{\mathrm{c}}$ and anti- $\mathrm{H}_{\mathrm{u}}$ respectively) were prepared as follows: The seeds were ground to a fine powder and the meal was mixed with saline in a ratio of $1 / 4$ by weight, $(1 \mathrm{gm}$. of meal to $3 \mathrm{cc}$. of saline). The mixtures were agitated in a Kahn shaker for an hour. After settling overnight the mixtures were centrifuged for twenty minutes at $6,000 \mathrm{rpm}$. The supernatant fluid was then frozen and constituted the concentrated stock. These initial preparations of both extracts were used throughout the experiment. This was done to avoid changes in the potencies of the extracts which are introduced by the use of different seed lots. The usual deviations observed in the preparation of several stocks were also avoided by the use of only one preparation. The agglutination titers (after centrifugation) of these stock fluids tested with the same control type 0 cells are shown in Table I.

\section{TABLE I}

Agglutination Titers of the Anti-H Reagents Extracted from Cytisus sessilifolius $\left(A n t i-H_{\mathrm{c}}\right)$ and Ulex europaeus $\left(A n t i-H_{\mathrm{u}}\right)$ Tested with the Same O Red Cells.

\begin{tabular}{lccccccc}
\hline Extract & \multicolumn{8}{c}{ Agglutination Titers } \\
Dilutions & $1 / 2$ & $1 / 4$ & $1 / 8$ & $1 / 16$ & $1 / 38$ & $1 / 64$ & $1 / 128$ \\
\hline Anti-H $_{c}$ & 4 & 4 & 4 & 4 & 3 & 3 & - \\
Anti-Hu $_{u}$ & 4 & 4 & 3 & 3 & 2 & 1 & - \\
\hline
\end{tabular}

In the agglutination titers shown in Table $I$, as well as in the saliva inhibition titers, it has been observed that the Cytisus extract exhibits a threshold effect. That is, upon progressive dilutions, the anti- $\mathrm{H}_{\mathrm{c}}$ loses potency abruptly instead of gradually, as does the Ulex extract. In the inhibition tests, this holds true regardless of whether the salivas have been titrated against constant dilutions of anti- $\mathrm{H}_{\mathrm{c}}$, or a constant saliva concentration has been tested against varying dilutions of the extract.

For testing, the anti- $\mathrm{H}_{\mathrm{c}}$ and anti- $\mathrm{H}_{\mathrm{u}}$ stocks were diluted to $1 / 50$ and $1 / 20$ respectively. At these dilutions, both the reagents had been inhibited by $1 / 512$ dilution of the control $\mathrm{O}$ saliva.

Salivas: Saliva specimens were collected at random from fifty individuals of each of five $A B O$ blood groups $\left(A_{1}, A_{2}, O, B\right.$, and $\left.A B\right)$ with a total number of 250 specimens titrated and tested. The $A B$ group consisted of $42 A_{1} B$ and $8 A_{2} B$.

Methods:

(1) Two-fold dilutions of each saliva were prepared in duplicate in $.85 \%$ saline solution. The dilutions in each set ranged from $1 / 2$ to $1 / 102$ (ten tubes with one drop of diluted saliva in each). 
(2) One drop of $1 / 50$ dilution of anti- $\mathrm{H}_{\mathrm{c}}$ was added to each tube of the first set and one drop of anti- $\mathrm{H}_{\mathrm{u}}$ at $1 / 20$ to each of the tubes of the second set. They were then left to incubate for twenty minutes at room temperature.

All fluids were delivered to the tubes using a single pipette, made by imbedding a steel, flat tip, 18 gauge needle in a glass tube. In this manner, although drop size was not measured, it could be assumed that there would be very little variation in volume between tubes.

(3) One drop of $2 \%$ suspension of "control" $O$ red cells were added to each of the twenty tubes and incubated for twenty additional minutes.

(4) After centrifugation for fifteen seconds at $2,000 \mathrm{rpm}$. the tubes were read, and the inhibition titers were recorded. For the anti- $\mathrm{H}_{c}$, the dilution which gave a reaction of 2 or more was taken as the end point. Any "+" agglutination was taken as the end point for the anti- $\mathrm{H}_{\mathrm{u}}$. These different end points were chosen because of the threshold demonstrated in the inhibition of the Cytisus extract.

From time to time during the experiment, samples from both reagents were tested against the control $O$ saliva to determine the repeatability of the test. At no time did the scores for the control saliva vary by more than one dilution from any other score in the repeatability series.

\section{Results and Discussion}

The results of the inhibition tests are summarized in Table II and Figure 1. For convenience in the computations of the means and the standard deviations, the saliva dilutions were coded with scores from 1 to 11 . Score " 1 " represents the $1 / 2$ titer, 10 the $1 / 1024$ and score 11 was substituted for titers of over $1 / 1024$.

An examination of the means of the rows in Table II indicates that there is considerable difference between blood groups in the potency of the $H$ substance found in salivas. $O$ salivas demonstrate the highest potency of substance, with $A_{2}, A_{1}, B$ and $A B$ following in that order. This relationship in strength of $H$ is noticed independently of the type of anti-H used. However, even though the trend is the same with both reagents, there is much variability between groups with respect to $H_{c}$ and $H_{u}$. (These last notations represent the reactivity of a saliva to each of the specific lectins, anti- $\mathrm{H}_{\mathrm{c}}$ and anti- $H_{u}$.) The variation in the potency of the $H$ substance is mainly due to the extreme types, $O$ and $A_{2}$ on one hand and $B$ and $A B$ on the other. The $O$ and $A_{2}$ salivas seem to inhibit the anti- $H_{u}$ better than they do the anti- $H_{c}$. The contrary is observed in the behaviour of $B$ and $A B$ salivas, which inhibit the anti- $H_{c}$ better than they do the anti- $H_{u}$. The components of Table II have been arranged in the form of frequency histograms and are presented in Fignre 1. Start- 

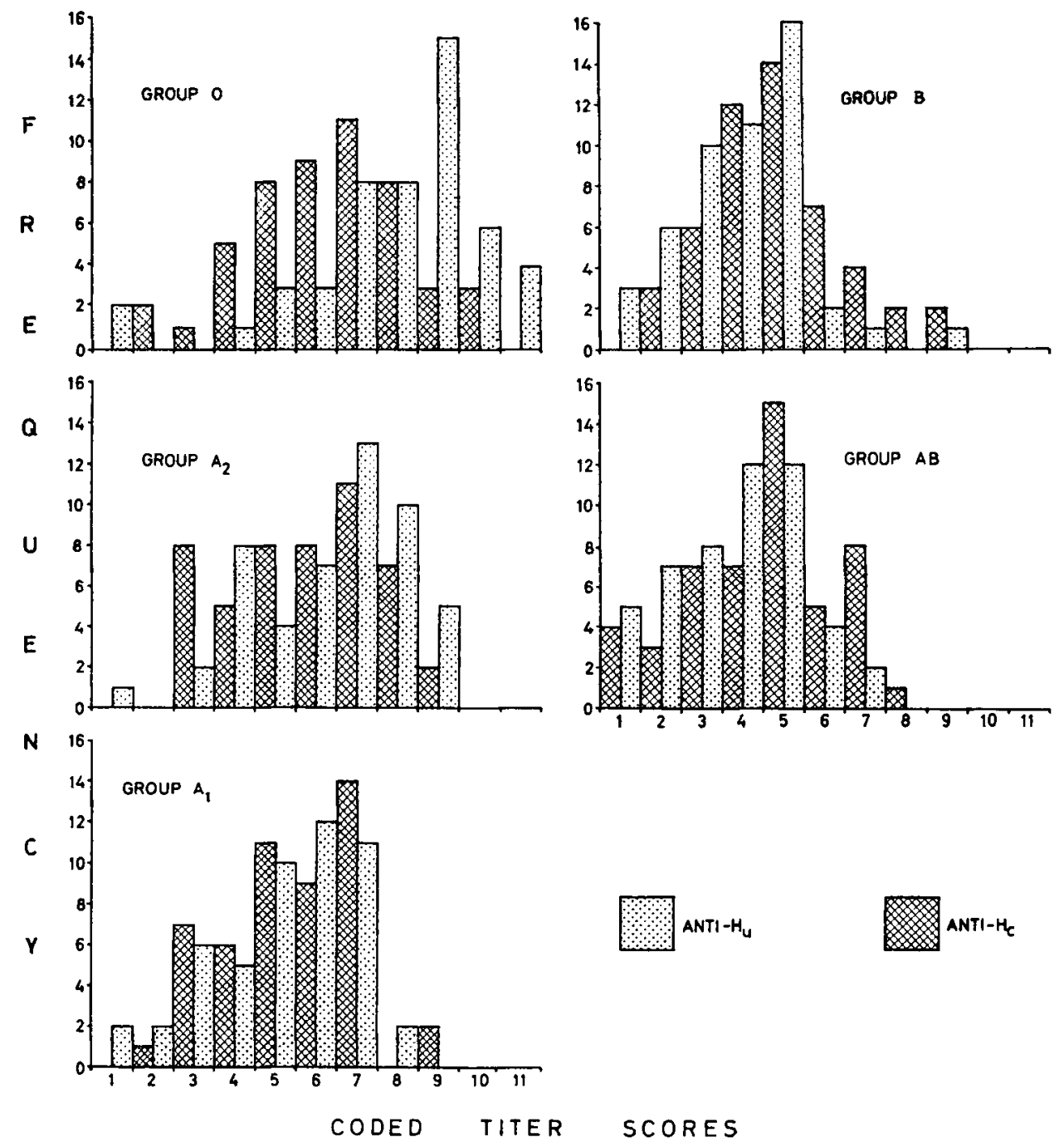

Fig. 1. Frequencies of the inhibition titer scores of salivas from persons of the several blood groups.

ing from the distributions of the $O$ group on top, down to the $A B$ at the bottom; note the tendency for a shift of the modes from higher $\mathrm{H}$ titer to lower. However, the changes in the reaction with the anti$\mathrm{H}_{u}$ seem to be more variable than the ones with anti- $\mathrm{H}_{\mathrm{c}}$. Thus, the mean titer of the $H_{u}$ is higher than that of $H_{c}$ in the groups of high 
340 Plato, Gershowitz, Specific Differences in the Inhibition Titers of

TABLE II

Frequencies of the Inhibition Titers of Saliva of the Five Blood Groups, Tested with Both Ulex europaeus and Cytisus sessilifolius.

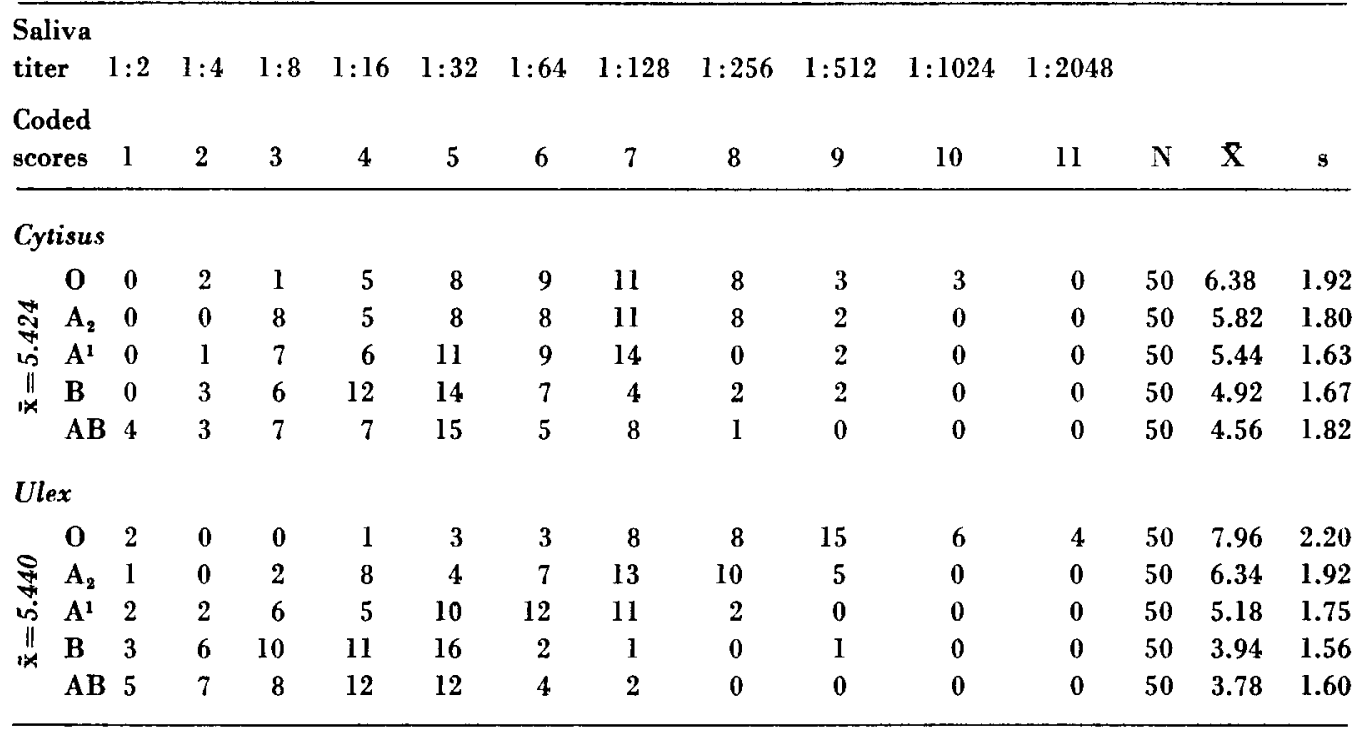

$H$ secretion ( $O$ and $A_{2}$ ) on one hand, while on the other hand it is less than the mean titer of $H_{c}$ in the groups with low $H$ secretions ( $B$ and $A B)$. This bi-directional change in potency can hardly be attributed to a quantitative difference between the two anti-H reagents, nor can it be attributed to quantitative variation of $\mathrm{Le}^{\mathrm{b}}$, with which anti-H may react, among the salivas. Mere quantitative variation would not have resulted in abi-directional change of potency. It would seem that the two anti-H reagents vary in specificity not only from group to group, but also within groups. Their differences in specificity may also be demonstrated by comparing the inhibition titer of each saliva against both of the reagents (Figures 2 and 3 and Table III).

Each saliva was titrated against constant dilutions of both anti- $\mathrm{H}_{\mathrm{c}}$ and anti- $\mathrm{H}_{\mathrm{u}}$ and the respective inhibition titers were recorded in scatter diagrams (Figure 2) for each blood group separately. If both reagents were equipotent for the same salivas, then the frequencies should be distributed along the $45^{\circ}$ diagonal as is the case in the group $A_{1}$. Concentrations of points above the regression line denote 


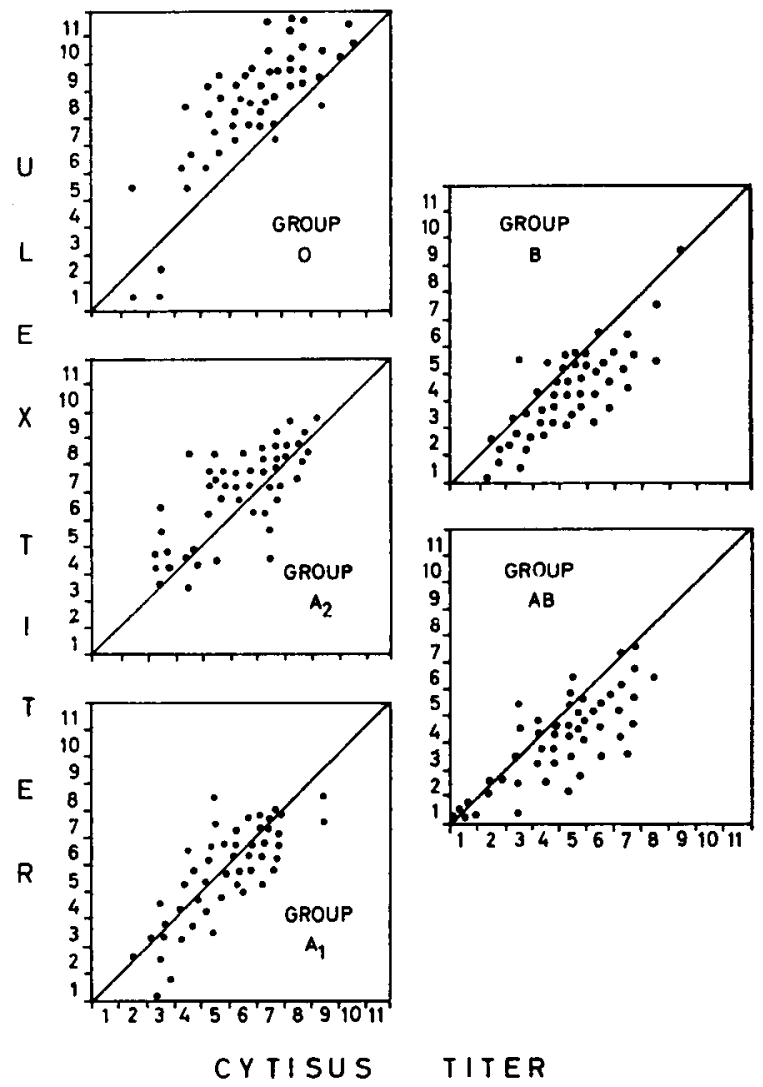

Fig. 2. Scatter diagrams of the inhibition titer scores for each blood group. Salivas were titrated against a constant dilution of Cytisus and Ulex extracts.

higher potency of $H_{u}$ whereas the concentrations below the line indicate that $\mathrm{H}_{\mathrm{c}}$ is more potent.

The comparisons presented in Table III were again based on inhibition titers of the anti- $\mathrm{H}_{c}$ and anti- $\mathrm{H}_{u}$. In this case, however, the actual difference in titer for each saliva was studied. Thus, if for the same specimen the inhibition titers were identical, the score zero $(0)$ was assigned. For salivas in which the titer of $H_{c}$ was higher than $H_{u}$ by two dilutions, the score two (2) was placed under Cytisus on the right side of the zero. If $\mathrm{H}_{\mathrm{u}}$ was higher, the appropriate scores were entered at the left side of the zero. Again the scores were coded for convenience. This classification enables us to test for the significance 
342 Plato, Gershowitz, Specific Differences in the Inhibition Titers of

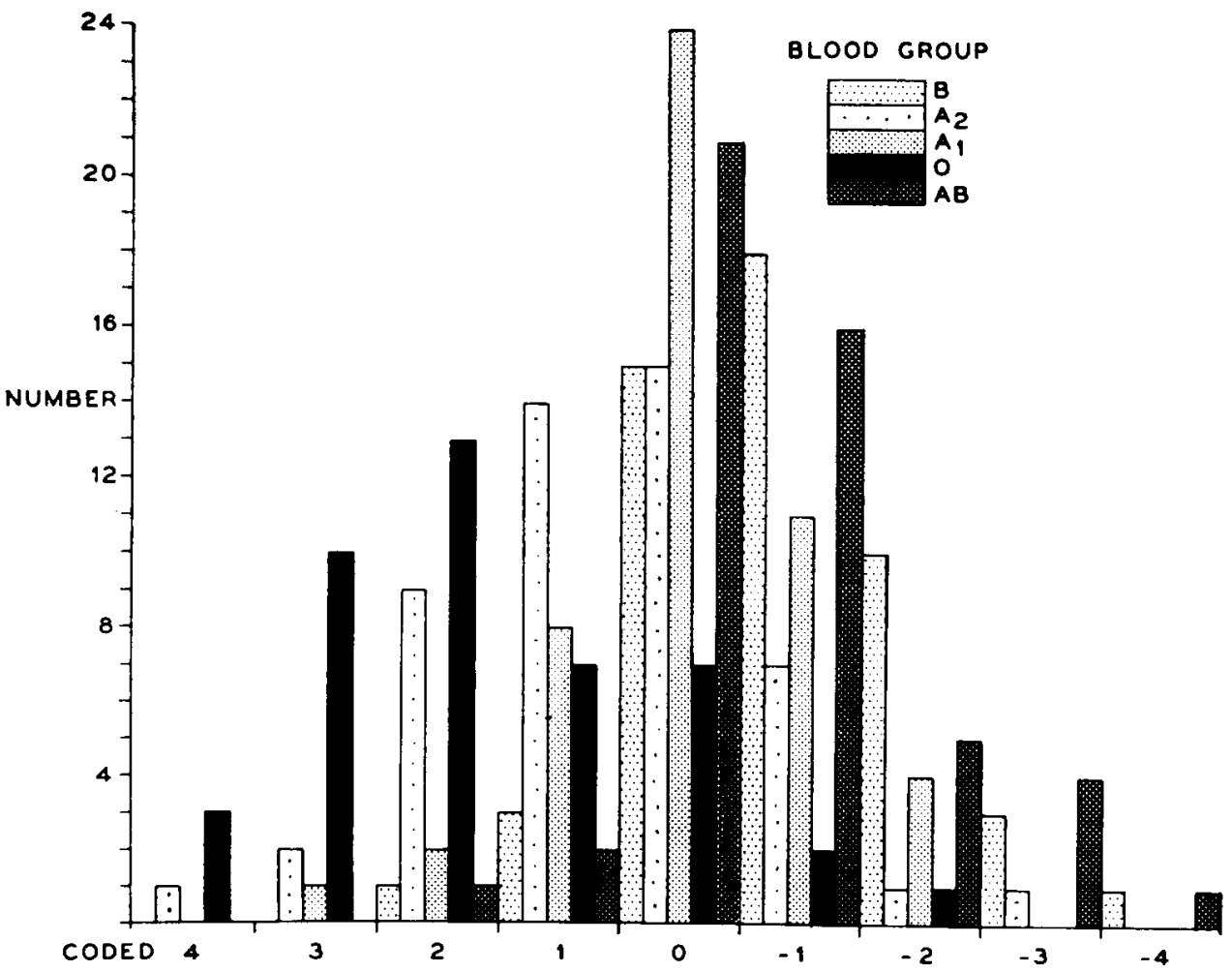

Fig. 3. Frequencies of the differences between the inhibition titers of Cytisus and Ulex extracts for all five blood groups.

\section{TABLE III}

Frequencies of the Differences between the Inhibition Titers of Anti-H and Anti-H $H_{\mathrm{u}}$ for All Five Blood Groups

\begin{tabular}{lrrrrrrrrrrrc}
\hline & \multicolumn{1}{c}{ Ulex } & \multicolumn{1}{c}{ Cytisus } & Mean & $\mathrm{s}_{\mathrm{d}}$ & $\mathbf{t}$ \\
Difference (d) & 4 & 3 & 2 & 1 & 0 & 1 & 2 & 3 & 4 & $\mathrm{dev}$. & & $(\overline{\mathrm{d}-\mathrm{d}})$ \\
Coded scores & 4 & 3 & 2 & 1 & 0 & -1 & -2 & -3 & -4 & $(\overline{\mathrm{d}})$ & & \\
\hline $\mathbf{s} \overline{\mathrm{d}}$
\end{tabular}

* significance at the .01 level. 
of the differences in the specificities of the anti- $\mathrm{H}_{c}$ and the anti- $\mathrm{H}_{u}$ reagents within blood groups and among different blood groups.

The first test is carried out by using the statistic " $t$ ", where $t=$ $(\bar{d}-d) / s \bar{d}$, and assuming that the two reagents have similar titers when tested with the same saliva. In the above formula $\bar{d}$ is the mean difference between the two reagents within groups; $d$ is the expected difference, which under the hypothesis should be zero, and $s_{d}$ is the standard error of the mean difference, which is found by $s \bar{d}=s / \sqrt{n}$, where $s$ stands for standard deviation. The results of the " $t$ " tests give evidence for rejecting the hypothesis of homogeneity (signifcance at the $1 \%$ level), between the two reagents for the $A_{2}, 0$, the $B$ and the $A B$ salivas. On the other hand, their differences were not significant within the $A_{1}$ saliva group. More specifically, the potencies of the $H_{c}$ and $H_{u}$ are similar in the salivas of group $A_{1}$. The $H_{u}$ potency is seen to be significantly higher in $A_{2}$ and $O$, whereas $H_{c}$ is significantly more potent in the salivas of groups $B$ and $A B$. The frequencies observed in Table III, have been arranged in the form of histograms in Figure 3.

TABLE IV

Analysis of Variance of the Differences in the Inhibition Titers of Anti- $H_{\mathrm{c}}$ and Anti- $H_{\mathrm{u}}$.

\begin{tabular}{lrrr}
\hline Source & d.f. & s.sq. & m.sq. \\
\hline Total & 249 & 574 & - \\
Between groups & 4 & 200 & 50.00 \\
Within groups & 245 & 374 & 1.53 \\
\hline
\end{tabular}

$$
\begin{aligned}
& " F "=\frac{50.00}{1.53}=32.62 * *, \text { d.f. } 4,245 . \\
& * * \text { significance at the } .01 \text { level. }
\end{aligned}
$$

To test whether the differences among the various blood groups are due to certain peculiarities of the reagents involved or due to chance variations, an analysis of variance was performed (Table IV). The "F" test is highly significant (at the $1 \%$ level), which gives evidence that the differences between the various blood groups are not due to chance, but that the activity of the two anti-H reagents actually varies from group to group. 
344 Plato, Gershowitz, Specific Differences in the Inhibition Titers of

It is reasonable to expect that the threshold effect observed in the activity of anti- $H_{c}$ would have decreased the actual titer of the saliva. That is, a specimen scored 7 , with an agglutination reading of 3 , might have been scored, in the absence of the threshold phenomenon, 8 or 9 with agglutination readings of 2 or 1 . This effect, however, does not alter the fact that there is variability between the extracts; while the threshold increases the variation between the inhibition titers of the two anti-H reagents in the salivas of $\mathrm{O}$ and $\mathrm{A}_{2}$ individuals, it tends to decrease it in the salivas of $B$ and $A B$ blood groups.

\section{TABLE V}

Inhibition Tests for Complimentarity of Anti-H Reagents Extracted from Three Different Seeds.

\begin{tabular}{|c|c|c|c|c|c|}
\hline \multirow{2}{*}{\multicolumn{2}{|c|}{$\begin{array}{c}\text { Neutralized reagent } \\
\text { and dilution }\end{array}$}} & \multirow{2}{*}{\multicolumn{2}{|c|}{$\begin{array}{l}\text { Added reagent } \\
\text { and dilution }\end{array}$}} & \multicolumn{2}{|c|}{ Saliva Dilutions } \\
\hline & & & & $1: 6$ & $1: 7$ \\
\hline Anti- $H_{c}$ & $(1: 4)$ & \multicolumn{2}{|c|}{ none } & 0 & + \\
\hline Anti- $\mathbf{H}_{\mathbf{c}}$ & $(1: 4)$ & Anti-He & $(1: 4)$ & 4 & \\
\hline Anti-H & $(1: 4)$ & Anti- $H_{u}$ & $(1: 2)$ & 0 & \\
\hline Anti-H $\mathrm{H}_{\mathrm{c}}$ & $(1: 4)$ & Anti- $H_{t}$ & $(1: 16)$ & 2 & \\
\hline Anti-H $H_{u}$ & $(1: 2)$ & \multicolumn{2}{|c|}{ none } & 0 & \pm \\
\hline Anti-H $H_{u}$ & $(1: 2)$ & Anti- $H_{u}$ & $(1: 2)$ & + & \\
\hline Anti-H & $(1: 2)$ & Anti-He & $(1: 4)$ & 0 & \\
\hline Anti- $\mathrm{H}_{\mathrm{u}}$ & $(1: 2)$ & Anti- $H_{t}$ & $(1: 16)$ & 0 & \\
\hline Anti-H $\mathrm{H}_{\mathrm{t}}$ & $(1: 16)$ & \multicolumn{2}{|c|}{ none } & 0 & \pm \\
\hline Anti-H $H_{t}$ & $(1: 16)$ & Anti-H $H_{t}$ & $(1: 16)$ & + & \\
\hline Anti- $H_{t}$ & $(1: 16)$ & Anti- $H_{c}$ & $(1: 4)$ & 1 & \\
\hline Anti-H $\mathrm{H}_{\mathrm{t}}$ & $(1: 16)$ & Anti-Hu & $(1: 2)$ & 0 & \\
\hline
\end{tabular}

Finally, a neutralization test was carried out. In this experiment a third anti-H reagent was included (Tetragonolobus purpureus), anti- $\mathrm{H}_{t}$. The dilutions of $1 / 4$ for anti- $\mathrm{H}_{c}, 1 / 2$ for anti- $\mathrm{H}_{\mathrm{u}}$ and $1 / 16$ for anti- $H_{t}$ were selected, since, at these dilutions, all three were inhibited by the type $\mathrm{O}$ saliva at $1 / 6$ dilution, but all three demonstrated only partial inhibition when the saliva was diluted to $1 / 7$ (Table V). Four tubes of each reagent were neutralized with the same saliva at a dilution of $1 / 8$. After neutralization, the first tubes of each reagent 
served as the negative controls. In the second tube of each set, an additional drop of the same anti-H extract was added. In the remaining two tubes, one drop of either of the other two reagents was placed. The results of the neutralization tests recorded in Table IV support the statistical findings, that the anti- $\mathrm{H}_{c}$ and anti- $\mathrm{H}_{u}$ are not testing for the same substance.

Note that neutralization with anti- $\mathrm{H}_{\mathrm{c}}$ is apparently complete as far as the recovery of additional anti- $H_{c}$ is concerned but is not complete as far as the added anti- $\mathrm{H}_{\mathrm{u}}$ is concerned. (We say "apparently" complete, for it is not certain how the threshold effect evident in the use of Cytisus may affect such a neutralization experiment.) Neutralization with anti- $\mathrm{H}_{\mathrm{u}}$, on the other hand, inactivates the saliva's further neutralizing capacity only towards anti- $\mathrm{H}_{\mathrm{u}}$. The anti- $\mathrm{H}_{t}$ behaves, in all cases, as the anti- $\mathrm{H}_{\mathrm{c}}$. The reactions noted in the table support the statistical findings that anti- $\mathrm{H}_{c}$ and anti- $\mathrm{H}_{u}$ each sah a unique anti-H specificity.

Preliminary experiments with rabbit immune sera have demonstrated at least one antigenic difference between the two extracts. Rabbit anti-Cytisus inhibits the agglutination activity of Cytisus extract toward $O$ red blood cells but does not affect the activity of Ulex extract. Rabbit anti-Ulex, however, inhibits both extracts. The relation of the agglutination inhibiting antibody to an antibody capable of inhibiting the saliva neutralizing capacity of the extracts awaits further investigation.

\section{Summary}

Test of two commonly used anti-H reagents (extracts from Cytisus sessilifolius and Ulex europaeus) demonstrated that group $O$ secretors have the highest titer of $H$ substance, with $A_{2}, A_{1}, B$, and $A B$ followhing in that order. $A$ comparison between the two extracts indicated that saliva belonging to persons of group $A_{1}$ have about the same amount of both $H_{u}$ and $H_{c}$ (substances inhibiting the Ulex and Cytisus extracts respectively), $A_{2}$ and $O$ groups have a much higher titer of $H_{u}$ substance than they do $H_{c}$, and $B$ and $A B$ groups demonstrate exactly the opposite qualities, with higher $H_{c}$ than $H_{u}$ titers. The most plausible explanation of the data seems to be that at least two different types of anti-H specifities are involved (anti- $\mathrm{H}_{c}$ and anti- $\mathrm{H}_{\mathrm{u}}$ ); these extracts, then, differ not only quantitatively, but qualitatively as well. 


\section{Résumé}

Les test pratiqués avec deux réactifs anti-H utilisés habituellement (extraits du Cytisus sessilifolius et de l'Ulex europaeus) ont démontré que les secréteurs du groupe $O$ possèdent le plus de substances $H$ et ensuite, par ordre décroissant, les secréteurs des groupes $A_{2}, A_{1}, B$ et AB.

Une comparaison établie entre les deux extraits a démontré que la salive des personnes de groupe $A_{1}$ a la même quantité de substances $H_{c}$ et $H_{u}$ (substances inhibant les extraits de Cytisus sessilifolius et d'Ulex europaeus respectivement), que la salive des personnes de groupe $A_{2}$ et $O$ ont une contenance plus grande en substance $H_{u}$, que la salive des personnes de groupe $B$ et $A B$ montre des propriétés exactement contraires avec une contenance plus forte en $H_{c}$ qu'en $H_{u}$. L'explication la plus plausible de ces résultats semble être le fait qu'il y a au moins deux spécificités différentes de l'anti-H (l'anti- $\mathrm{H}_{c}$ et l'anti- $\mathrm{H}_{\mathrm{u}}$ ). Ces extraits se différencient non seulement au point de vue quantitatif mais également au point de vue qualitatif.

\section{Zusammenfassung}

Untersuchungen mit zwei gebräuchlichen Anti-H-Reagentien (Extrakte von Cytisus sessilifolius und Ulex europaeus) zeigten, daß Sekretoren der Gruppe $O$ die höchsten H-Substanz-Titer aufweisen. Bei Sekretoren der Gruppen $A_{2}, A_{1}, B$ und $A B$ sind die Titer niedriger, wobei der H-Substanz-Gehalt von $\operatorname{den} A_{2^{-}}$zu den AB-Individuen progressiv abnimmt. Vergleichsuntersuchungen mit den beiden Extrakten zeigten, daß der Speichel von $A_{1}$-Personen etwa gleichviel $H_{u^{-}}$und $H_{c^{-}}$Substanz enthält ( $H_{u}$ hemmt Ulex; $H_{c}$ hemmt Cytisus).

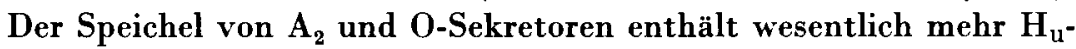
als $H_{c}$-Substanz. Der Speichel von B- und AB-Sekretoren enthält umgekehrt mehr $\mathrm{H}_{\mathrm{c}^{-}}$als $\mathrm{H}_{\mathrm{u}^{-}}$-Substanz. Die einfachste Erklärung dieser Befunde besteht in der Annhame, daß die Extrakte Anti-H«Antikörper» mit unterschiedlicher Spezifität enthalten. Die unterschiedliche Wirkung der Extrakte beruht somit nicht bloß auf quantitativen, sondern auf qualitativen Differenzen der aktiven Komponenten.

\section{References}

1. Bird, G. W. G.: Specific agglutinating activity for human red blood corpuscles in extracts of Dolichos biflorus. Curr. Sci. 20: 298-299 (1951). 
2. Bird, G. W. G.: Haemagglutinins in seeds. Brit. med. Bull. 15 (2): 165-168 (1959).

3. Boyd, W. C. and Reguera, R. M.: Hemagglutinating substances for human cells in various plants. J. Immunol. 62: 333-339 (1949).

4. Boyd, W. C. and Shapleigh, E.: Separation of individuals of any blood group into secretors and non-secretors by use of a plant agglutinin (lectin). Blood 9: 1195-1198 (1954).

5. Boyd, W. C. and Shapleigh, E.: Specific precipitating activity of plant agglutinins (lectins). Science 119: 419 (1954).

6. Cazal, $P$. et Lalaurie, $M .:$ Recherches sur quelques phyto-agglutinines spécifiques des groupes sanguins ABO. Acta haemat. 8: 73-80 (1952).

7. Glynn, L. E. and Holborow, E. J.: Distribution of blood-group substances in human tissues. Brit. med. Bull. 15 (2): 150-153 (1959).

8. McNeil, C.; Trentelman, E.; Kreutzer, V. and Fullmer, C.: Aberrant secretion of salivary A, B and $\mathrm{H}$ group substances in human beings. Amer. J. clin. Path. 28 (2): 145-151 (1957).

9. Renkonen, $K$. O.: Studies on hemagglutinins present in seeds of some representatives of the family of leguminoseae. Ann. Med. exp. Biol. Fenn. 26: 66-72 (1948).

Authorg' address: Dr. Ch. C. Plato and Dr. H. Gershowitz, University of Michigan Medical School, Department of Human Genetics, Ann Arbor, Mich. (USA) 